\title{
A MINIMAX INEQUALITY AND ITS APPLICATIONS TO VARIATIONAL INEQUALITIES
}

\author{
ChI-LiN YeN
}

In this paper we get a slight generalization of a $\mathrm{Ky}$ Fan's result which concerns with a minimax inequality. We shall use this result to give a direct proof for the existence of solutions of the following two variational inequalities:

$$
\inf _{w \in T y}\langle w, y-x\rangle \leqq h(x)-h(y) \text { for all } x \in X,
$$

and

$$
\sup _{w \in T x}\langle w, y-x\rangle \leqq h(x)-h(y) \text { for all } x \in X,
$$

where $T \subset E \times E^{\prime}$ is monotone, $E$ is a reflexive Banach space with its dual $E^{\prime}, X$ is a closed convex bounded subset of $E$, and $h$ is a lower semicontinuous convex function from $X$ into $R$.

In this paper we get a slight generalization of a Ky Fan's result [4] which concerns with a minimax inequality. We shall use this result to give a direct proof for the existence of solutions of the following two variational inequalities:

$$
\inf _{w \in T^{\prime} y}\langle w, y-x\rangle \leqq h(x)-h(y) \text { for all } x \in X,
$$

and

$$
\sup _{w \in T^{x}}\langle w, y-x\rangle \leqq h(x)-h(y) \text { for all } x \in X,
$$

where $T \subset E \times E^{\prime}$ is monotone, $E$ is a reflexive Banach space with its dual $E^{\prime}, X$ is a closed convex bounded subset of $E$, and $h$ is a lower semicontinuous convex function from $X$ into $R$. In fact, we show that under our condition the inequalities (1) and (2) are equivalent, that is, the sets of solutions are coincide.

Many results on these problems were done for the case that $T$ is a single-valued monotone operator of $E$ into $E^{\prime}$ with a continuity in some sense. One may see, for examples, C. Stampacchia [12] and J. L. Lions and G. Stampacchia [7], [8] for the case that $T$ is linear in a Hilbert space E, P. Hartman and C. Stampacchia [5] and G. Minty [9] for the case that $T$ is nonlinear in a Hilbert space $E$, and G. Minty [10], F. E. Browder [1], [2], L. Leray and J. L. Lions [6] and U. Mosco [11] for the case that $T$ is nonlinear in a Banach space $E$.

1. Preliminary. In this section we give some known defini- 
tions (e.g., see [4], [10]) which will be used in our paper.

Definition 1.1. Let $E$ be a topological vector space and $X$ a convex subset of $E$. A real-valued function $h$ on $X$ is said to be

(i) lower semicontinuous if for each $t$ the set

$$
\{x \in X: h(x) \leqq t\}
$$

is closed.

(ii) convex if for $x, y$ in $X$ and $0 \leqq r \leqq 1$ we have $h((1-r) y+$ $r \cdot x) \leqq(1-r) h(y)+r h(x)$.

(iii) concave if $-h$ is convex.

(iv) quasiconcave if for each $t$ the set

$$
\{x \in X: h(x)>t\}
$$

is a convex (or empty) subset of $X$.

Definition 1.2. Let $E$ be a Banach space with a dual space $E^{\prime}, X \subset E$, and $T \subset X \times E^{\prime}$.

(i) $T$ is said to be monotone on $X$ if for each $x \in X$, there is a $u \in E^{\prime}$ with $(x, u) \in T$ and for $\left(x_{1}, u_{1}\right),\left(x_{2}, u_{2}\right) \in T$ we have

$$
\left\langle u_{1}-u_{2}, x_{1}-x_{2}\right\rangle \geqq 0,
$$

where $\langle u, x\rangle$ is defined to be the number $u(x)$.

(ii) $T x$ is defined to be the set $\left\{u \in E^{\prime}:(x, u) \in T\right\}$ for all $x \in X$.

(iii) $T$ is said to be hemiclosed on $X$ if for each $y \in X, z \in X$ and $0<t<1$ the conditions $\left((1-t) y+t z, u_{t}\right) \in T$ and $w$ - $\lim u_{t_{n}}=u_{0}$ for some $t_{n} \downarrow 0$ imply that $\left(y, u_{0}\right) \in T$.

(iv) $T$ is said to be $l$-bounded on $X$ if for any two points $y, z$ in $X$ there is a $r>0$ such that the set $\left\{\alpha_{t}: 0<t \leqq r\right\}$ is bounded, where $\alpha_{t}$ denotes the infimum of $\|u\|$ for all $u \in E^{\prime}$ satisfying

$$
((1-t) y+t z, u) \in T \text {. }
$$

2. Fan's minimax inequality, In this section we give a minimax inequality which is a slight generalized form of Fan [4], the technique of the proof follows from Fan [4] which is based on the following Fan's lemma [3].

Lemma. Let $X$ be an arbitrary set in a Hausdorff topological vector space $E$. To each $x \in X$ let a closed set $G(x)$ in $E$ be given such that $G(x)$ is compact for at least one $x \in X$. If the convex hull of every finite subset $\left\{x_{1}, x_{2}, \cdots, x_{n}\right\}$ of $X$ is contained in the corresponding union $\bigcup_{i=1}^{n} G\left(x_{i}\right)$, then $\bigcap_{x \in I} G(x) \neq \phi$. 
THEOREM 1. Let $X$ be a compact convex subset of a Hausdorff topological vector space $E$. If $f$ and $g$ are real-valued functions on $X \times X$ with the following properties: on $X$,

(i) for each $x \in X, g(x, \cdot)$ is a lower semicontinuous function

(ii) for each $y \in X, f(\cdot, y)$ is a quasi-concave function on $X$,

(iii) $g(x, y) \leqq f(x, y)$ for all $(x, y) \in X \times X$,

then the minimax inequality

$$
\min _{y \in X} \sup _{x \in X} g(x, y) \leqq \sup _{x \in X} f(x, x)
$$

holds.

Proof. Let $t=\sup \{f(x, x): x \in X\}$. Without loss of the generality we may assume that $t<+\infty$. For each $x \in X$, let

$$
\begin{aligned}
& F(x)=\{y \in X: f(x, y) \leqq t\}, \\
& G(x)=\{y \in X: g(x, y) \leqq t\} .
\end{aligned}
$$

Then by (i), (ii) and (iii) we have that

(iv) $G(x)$ is a closed subset of the compact set $X$ and hence $G(x)$ is compact for all $x \in X$,

(v) for any finite subset $\left\{x_{1}, x_{2}, \cdots, x_{n}\right\}$ of $X$ we have

$$
\operatorname{conv}\left\{x_{1}, x_{2}, \cdots, x_{n}\right\} \subset \bigcup_{i=1}^{n} F\left(x_{i}\right),
$$

(vi) for each $x \in X, F(x) \subset G(x)$, respectively. It follows from (v) and (vi) that we have

(vii) for any finite subset $\left\{x_{1}, x_{2}, \cdots, x_{n}\right\}$ of $X$ we have

$$
\operatorname{conv}\left\{x_{1}, x_{2}, \cdots, x_{n}\right\} \subset \bigcup_{i=1}^{n} G\left(x_{i}\right) .
$$

It is due to the above lemma and the fact that (iv) and (vii) holds we have that $\cap\{G(x): x \in X\} \neq \phi$.

Let $y_{0} \in \cap\{G(x): x \in X\}$. Then

$$
g\left(x, y_{0}\right) \leqq t \quad \text { for all } x \in X,
$$

and our minimax inequality holds.

3. Applications to variational inequalities. In this section we make use of theorem 1 to prove the existence of solutions for the variational inequalities (1) and (2).

Theorem 2. Let E be a reflexive Banach space with dual space 
$E^{\prime}$ and $X$ a closed bounded convex subset of $E$. If $T \subset X \times E^{\prime}$ is monotone $D(T)=X, h: X \rightarrow R$ is a lower semicontinuous, convex function. Then there is a $y_{0} \in X$ such that

$$
\sup _{u \in T x}\left\langle u, y_{0}-x\right\rangle \leqq h(x)-h\left(y_{0}\right) \text { for all } x \in X .
$$

Proof. The monotonicity ensures that for $(x, u),(y, v) \in T$, we have $\langle u, y-x\rangle+h(y)-h(x) \leqq\langle v, y-x\rangle+h(y)-h(x)$. Define, the real valued-functions $f$ and $g$ on $X \times X$ by

$$
\begin{aligned}
& f(x, y)=\inf \{\langle v, y-x\rangle+h(y)-h(x): v \in T y\}, \\
& g(x, y)=\sup \{\langle u, y-x\rangle+h(y)-h(x): u \in T x\} .
\end{aligned}
$$

Since $h$ is convex, lower semicontinuous with respect to the norm topology on $X$ we see that $h$ is also lower semicontinuous with respect to the weak topology on $X$. Then $f$ and $g$ satisfy the conditions (i), (ii) and (iii) with the weak topology on $X$ in Theorem 1 , hence there is a $y_{0} \in X$ such that

$$
\sup _{x \in I} g\left(x, y_{0}\right) \leqq \sup _{x \in X} f(x, x)=0,
$$

or

$$
\sup _{u \in T x}\left\langle u, y_{0}-x\right\rangle \leqq h(x)-h\left(y_{0}\right) \quad \text { for all } x \in X .
$$

THEOREM 3. In addition to the assumption of Theorem 2, assume further that $T$ is hemiclosed and l-bounded. Then there is $a y_{0} \in X$ such that

$$
\inf _{v \in T y_{0}}\left\langle v, y_{0}-x\right\rangle \leqq h(x)-h\left(y_{0}\right) \quad \text { for all } x \in X
$$

Proof. By Theorem 2 there is a $y_{0} \in X$ with

$$
\sup _{u \in T_{x}}\left\langle u, y_{0}-x\right\rangle \leqq h(x)-h\left(y_{0}\right) \quad \text { for all } x \in X .
$$

For $x^{\prime} \in X$ and $1>r>0$, let $x_{r}=y_{0}-r\left(y_{0}-x^{\prime}\right)$. Then $v_{r} \in X$ and

$$
\left\langle u_{r}, y_{0}-x_{r}\right\rangle \leqq h\left(x_{r}\right)-h\left(y_{0}\right)
$$

for all $u_{r} \in T x_{r}$. The convexity of $h$ implies that

$$
h\left(x_{r}\right)-h\left(y_{0}\right) \leqq r\left(h\left(x^{\prime}\right)-h\left(y_{0}\right)\right) \quad \text { for all } 0<r<1 .
$$

Hence,

$$
\left\langle u_{r}, y_{0}-x^{\prime}\right\rangle \leqq h\left(x^{\prime}\right)-h\left(y_{0}\right) \quad \text { for all } r_{r} \in T x_{r} .
$$

It follows from the $l$-boundedness of $T$ that there 1 s a subsequence 
$\left\{r_{n}\right\}$ of $\{r\}$ such that $\lim r_{n}=0$ and $\left\{r_{r_{n}}\right\}$ has some weak limit $v_{0} \in E^{\prime}$. Moreover, by the hemiclosedness of $T$ that $v_{0} \in T y_{0}$ and

$$
\left\langle v_{0}, y_{0}-x^{\prime}\right\rangle \leqq h\left(x^{\prime}\right)-h\left(y_{0}\right),
$$

that is

$$
\inf _{v \in T y_{0}}\left\langle v, y_{0}-x^{\prime}\right\rangle \leqq h\left(x^{\prime}\right)-h\left(y_{0}\right) \quad \text { for all } x^{\prime} \in Y \text {. }
$$

REMARK. The inequality (1) always implies (2), but under the hypothesis of Theorem 3 we have shown that (2) implies (1) in Theorem 3. Hence the inequalities (1) and (2) are equivalent.

The author would like to express his gratefulness to the referee who pointed out a lot of misprints in the original copy of this paper.

\section{REFERENCES}

1. F. E. Browder, Nonlinear elliptic boundary value problems, Bull. Amer. Math. Soc., 117 (1963), 862-274.

2. - Nonlinear elliptic boundary value problems, II, Trans. Amer. Math. Soc., 117 (1965), 530-550.

3. K. Fan, A generalization of Tychonoff's fixed point theorem, Math. Ann., 142 (1961), 305-310.

4. - A min-max inequality and applications, Inequalities III Shisha, ed., Academic Press (1972), 103-113.

5. P. Hartaman and G. Stampacchia, On some nonlinear elliptic differential functional equations, Acta Math., 115 (1966), 271-310.

6. J. Leray and J. L. Lions, Quelques resultats de Visik sur les problemes elliptic nonlineaires par les methodes de Minty-Browder, Bull. Soc. Math. France, 93 (1965), 97-107.

7. J. L. Lionsand and G. Stampacchia, Inequations variationnelles non coercives, Compt. Rend. Acad. Sci. Paris, 261 (1967), 25-27.

8. - Variational inequalities, Commun. Pure Appl. Math., 20 (1967), 493-519.

9. G. Minty, Nonotone (non linear) operators in Hilbert space, Duke Math. J., 29 (1962), 341-346.

10. - On a "monotonicity" method for the solution of non linear equations in Banach spaces, Proc. Natl. Acad. Sci. U.S.A. 50 (1963), 1038-1041.

11. U. Mosco, Convergence of convex sets and od solutions of variational inequalities, Adv. Math., 3 (1969), 510-585.

12. G. Stampacchia, Formes bilineaires coercitives sur les ensembles convexes, Compt. Rend. Acad. Sci. Paris, 258 (1964), 4413-4416.

Received June 20, 1980 and in revised form October 27, 1980. This work was supported by NSC, Republic of China.

National Taiwan Normal University

TAIPEI, TAIWAN

Republic of China 
